\title{
CREATING PERCEPTUAL UNCERTAINTY IN AGENT-BASED MODELS WITH SOCIAL INTERACTIONS
}

\author{
Ethan O. Grantham \\ Departments of Computer Science \& Psychology \\ Furman University \\ 3300 Poinsett Hwy \\ Greenville, SC 29613, USA \\ ethan.grantham@furman.edu \\ Philippe J. Giabbanelli \\ Department of Computer Science \& Software Engineering \\ Miami University \\ 510 E. High St. \\ Oxford, OH, USA \\ giabbapj@miamioh.edu
}

\begin{abstract}
Simple Agent-Based Models (ABMs) of social influence assume that agents can perfectly discriminate between the actions of their peers or implicitly agree on a population-wide definition of states such as 'healthy' or 'unhealthy'. In reality, mistakes are a part of being human and incorrectly estimating the state of a peer is no exception. For instance, experimental studies show that whether we find a food 'healthy' also depends on the body size of those who eat it. In this paper, we introduce norm perception errors in models from common application areas of ABMs, namely computational sociology and public health. Using simulations, we examined whether an increase in perceptual uncertainty changed the outcome of the models reported by their authors. We found that the effect depends on the model: even frequent incorrect judgments by the agents had no effect in an eating model whereas correlations were statistically significant in a segregation model.
\end{abstract}

Keywords: Agent Based Models, Cognition, Health Behavior, Perception, Uncertainty

\section{INTRODUCTION}

As a result of rapid growth in computational power and wider availability of large data sets, experts have increasingly employed computational approaches to support decision-making activities that are affected by social interactions. Agent-based simulation models (ABMs) represent an especially promising framework for exploratory and predictive models in a variety of contexts. Compared to traditional models that use differential equations to represent populations, ABMs are advantageous for embodying systems with heterogeneous agents, local rules, and discrete rather than continuous representations of space, time, and state (Wilensky 
and Rand 2015, Railsback and Grimm 2010). Such portrayals often fit better with systems that have meaningful differences between individuals who react to local, rather than global, information. Representing details at a micro level enables modelers to create models that are more accurate and/or support a wider range of decision-making activities, but comes at the price of a heavy demand for data and computational resources (Railsback and Grimm 2010). From epidemiological models of H1N1 and HIV (dos Santos and Coutinho 2001) to city planning models of bar districts (Spicer et al. 2012), ABMs allow experts to test theories, explore different scenarios, and develop interventions for complex problems. This level of experimentation is particularly useful as decision-makers can use ABMs as a virtual environment to test several scenarios rather than dealing with the immediate real-life consequences of a sub-optimal choice (Brashears et al. 2019). Depending on the purpose of the model, fidelity to the real inner-workings of the system can be crucial (Ferreira 2019). Such fidelity partly relies on the existence of rules, which can be derived from highquality datasets for empirically-grounded models (Wilensky and Rand 2015, Giabbanelli 2019) or built on theories through collaborative efforts between simulationists and experts in the application domain (Ferreira 2019, Brashears et al. 2019). In this paper, we focus on the rules governing the information that agents use when making decisions based on social interactions.

ABMs of human behaviors must operationalize one essential yet complex question: why do people behave the way they do in a given context? A person's actions are shaped in part by interactions with the environment, which includes the social context of interactions with peers. While it is relatively straightforward to empirically observe and thus model some of these interactions (e.g., who talks with whom), it is less obvious what a person takes away from an interaction. In particular, humans only take in a small portion of the information available in their environment (Burden and Savin-Baden 2019) and potentially make errors in memory encoding or retrieval. As an example, consider vision: the center of our vision is very high resolution, but on the periphery the finer details become blurry. Similarly, our perceptual attention is limited by our cognitive capacity; we are very effective at tasks that capture our narrow attention, but this requires filtering out information that is irrelevant (Ferreira 2019). This notion that agents are cognitively limited rather than omniscient is best known as 'bounded rationality' (Simon 1957). In order to account for these limitations, we have developed shortcuts that allow us to determine what information is the most important while ignoring details we determine irrelevant. Using our beliefs and understanding of the world to interpret information, we are constantly reevaluating our goals and making decisions (Nyblade, O'Mahony, and Sieck 2019). While such a belief system can guide the way people view the world, it is important to note that it comes at a cost; beliefs can lead people to make incomplete or incorrect conclusions about the world through their biases (Nyblade, O’Mahony, and Sieck 2019, Tversky and Kahneman 1974). This means that instead of gathering all the available information about a situation, people may sometimes just make their best guess, which can lead to errors in judgment.

While many of our actions come from an internal belief-desire system, they are also driven by external influences coming from family, friends, and peers (Kadushin 2012b). In other words, we do act on our own beliefs, but social groups exchange information that can result in dynamic, group-wide beliefs and norms (Nyblade, O'Mahony, and Sieck 2019, Kadushin 2012a). The impact of social norms has long been documented and studied by social scientists (Asch 1955, Russell 2011). Given that (i) individuals can erroneously process information and that (ii) information driving the behavior of an individual also comes from peers, it follows that individuals can make errors in judgment about their environment, specifically about the behavior that they attribute to their peers. Judgment errors are inconsistently addressed in ABMs (Evans 2012), particularly when it comes to social interactions contributing to decision-making. Some models entirely omit the notion of errors in judgment (Giabbanelli et al. 2012, Zhang et al. 2014), while others may include an all-encompassing error term or use more sophisticated techniques such as using fuzzy sets "in the functions that model the evolution of individual change of mind [or] the relationships among individuals in a social network" (Hassan et al. 2010). 
Our overarching goal is to examine how the conclusions reached using a model may change when increasing the realism of peer influences via errors in judgment. To accomplish this objective, we identify and implement representative models which assume perfect information processing, verify that our baseline implementation matches the authors' results, and gradually increase the amount of errors in judgments (thus representing increased uncertainty) such that the model no longer assumes certainty. To avoid limitations inherent to using a single model, we selected two models from common application areas of ABMs, namely computational sociology and public health.

The remainder of the paper is structured as follows. In section 2, we summarize the use of ABMs in computational sociology and public health, together with the purpose and rules of the selected model in each area. Section 3 introduces our implementations and experimental design, that is, how the selected models are modified to account for norm perception errors. Section 4 presents the results of our experiments, which are discussed in section 5 within the context of decision-making models.

\section{BACKGROUND}

\subsection{Eating model}

Computational approaches have become increasingly sought in public health problems (Mabry et al. 2010, Maglio and Mabry 2011). This heightened demand has led to a burgeoning area of research coined 'systems modelling', in which conceptual or computational models have captured systems or subsystems relevant to public health such as physiological systems, social systems, or urban systems (Carey et al. 2015). ABMs already had an established track-record in the 2000's to model infectious diseases, with examples such as the Global-Scale Agent Model for disease outbreak (Parker and Epstein 2011). In the 2010's, ABMs also became an important tool for health behaviors that shape chronic conditions (Nianogo and Arah 2015, Badham et al. 2018) such as obesity, modeled through subsystems such as eating behaviors (Khademi et al. 2018, Giabbanelli and Crutzen 2017). A typical assumption in models for obesity (Giabbanelli et al. 2012, Bahr et al. 2009) or drinking (Dabbaghian et al. 2011, Giabbanelli and Crutzen 2013, Spicer et al. 2012) is that interactions between two agents occur without biases or perceptual mistakes such as misidentifying the group to which a peer belongs. Our first model thus draws from this application context by simulating eating behaviors. This model was proposed in (Dabbaghian et al. 2012) to represent the eating behaviors of North-American high-school students, with a focus on the effects of social influence on the food agents eat for lunch. Agents transition between 4 states, based on food choice:

$\begin{array}{ll}\text { BH } & \text { Bringing healthy food from home } \\ \text { BU } & \text { Bringing } \underline{\mathbf{u}} \text { nhealthy food from home } \\ \text { PH } & \text { Purchasing healthy food from school vendors } \\ \text { PU } & \text { Purchasing } \underline{\mathbf{u}} \text { nhealthy food from school vendors }\end{array}$

Upon initialization, the agents are randomly assigned to a cell in a $40 \times 40$ grid, such that the grid is composed of $25 \%$ of agents of each type ( $25 \% \mathrm{BH}, 25 \% \mathrm{BU}$, etc.). During each time step, each agent is influenced by their neighboring agents using $\alpha$ to represent the influences on students bringing or purchasing food and $\beta$ to represent the influences on students consuming healthy or unhealthy food. Positive $(P)$ and Negative $(N)$ parameters were chosen to control the values of $\alpha$ and $\beta$, such that a higher $P$ encouraged students to eat healthy food and a higher $N$ encouraged them to eat unhealthy food. All transitions between states are based on deterministic rules that specify a threshold where the influence of the neighbors on the agent overcomes their state inertia and the agent transitions from one state to the next. The model also included a purchasing power parameter that controlled agents' ability to transition to purchasing lunch, but this parameter was set to $100 \%$ for all of our simulations, effectively making it a non-factor. For a more detailed explanation of the model, see the original article (Dabbaghian et al. 2012). 


\subsection{Schelling Model of Housing Segregation}

Our second area of application is computational sociology, also known as social simulation. This area has a rich history spanning decades, which has been reviewed in detail (Bianchi and Squazzoni 2015, Klein et al. 2018). The Schelling model is an established model in this area. Although it was developed fifty years ago (Schelling 1971), it continues to be used in sociological research as a guiding example (ChattoeBrown 2013). Its rules or initial configurations are altered through the many extensions that appear every year (Echzell et al. 2019, Urselmans and Phelps 2018, Immorlica et al. 2017). The Schelling model thus constitutes the second model revisited in our study.

The Schelling model was the first to test key concepts in influencing individual relocation choices, which ultimately shape the overall spatial distribution of populations in suburbia and cities (Schelling 1971). Similarly to the previous model, the space is represented as a grid. In the Schelling model, each cell contains up to one agent. Agents can be in one of two states (referred to as zeros and stars in the original), meant to represent two different social or ethnic groups that live in the same area. The synchronous update at each time step allows agents to change their position. Members of each group will move to nearby empty spaces until the surrounding 8 agents (Moore neighborhood) are sufficiently similar to them. The agents' tolerance is controlled by a deterministic parameter, and can be represented in terms of an integer or a ratio. For example, agents could be satisfied (thus they stay in the same spot) if they have at least 2 neighbors that are the same state as them, or they could only be satisfied if $50 \%$ of their neighbors have the same state.

Notable probabilistic parameters that control the initialization of the model include density of agents in the grid and initial minority/majority ratio. For example, if the density was set to $70 \%$ and the minority/majority ratio was 1:3 (25\% minority, $75 \%$ majority), the model would follow the following procedure. For each cell of the grid, with $70 \%$ chance, add one agent in the cell; otherwise move to the next cell. If an agent was added, with $25 \%$ chance assign it type 0 ; otherwise assign it type 1 .

\section{METHODS}

\subsection{Implementations and verification}

Our implementations are available on the third-party Open Science Framework repository at https://osf.io/ n6pja/. Models were implemented in Python 3.7, using a framework package designed for ABMs called Mesa (Masad and Kazil 2015). Mesa provides built-in classes and functions to accelerate the development of ABMs. In line with our previous work (Giabbanelli et al. 2019), we systematically engage in, and describe the process for, verification of our implementations. Verification is essential to appropriately attribute any changes seen in the outcome of our experiments to challenging the models' assumption of perfect judgment, rather than a fundamental difference between our replicated version and the original.

We verified our implementation of the eating model by replicating the results displayed in the original article. These results were produced with a single run of the model, although there is randomness in its initialization. Our replications confirm that the original behavior is qualitatively replicated (Figure 1). Specifically, we see that the model stays at equilibrium when positive and negative influences are equal (Figure 1-a-b). At a $10 \%$ increase in positive eating influences, the populations that chose healthy foods dominate the unhealthy populations (Figure 1-c-d). At a $20 \%$ increase, this gap has widened further (Figure 1-e-f). On average, the outcomes from our run differed by $9.22 \%$ from the run shown in the original paper for the stochastic model.

Figure 2 shows our replication of an experiment in which negative influences are varied while keeping positive ones constant, thus doing the opposite of Figure 1. Again, we can see that our replicated model behaves similarly to the original. In sum, within the limitations of the original article, we have verified that our replicated model responds the same way to changes in the main influence parameters, and this provides an established baseline for the behavior of the model before we add errors to the agents' judgments. 

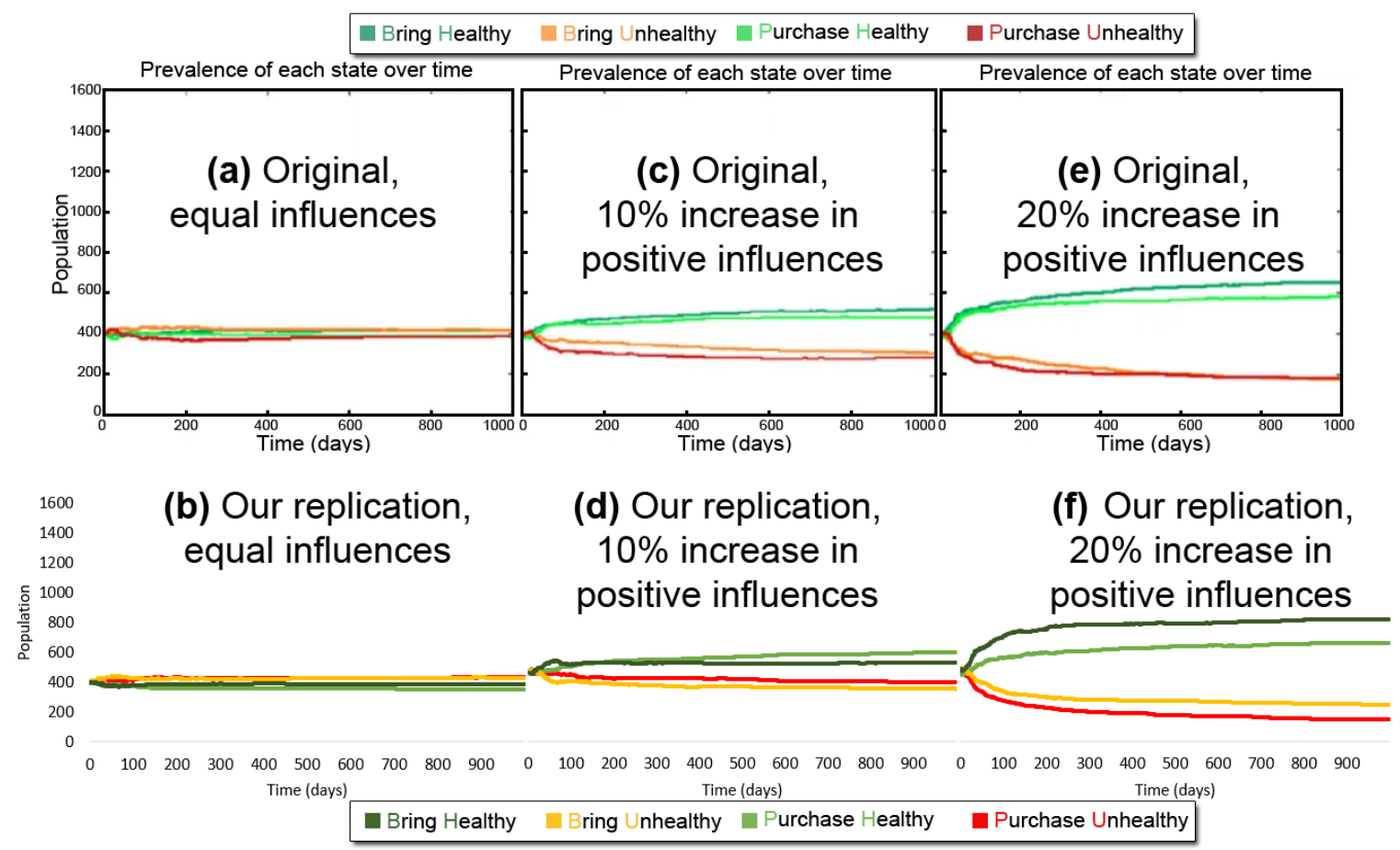

Figure 1: Top shows original results (a-c-e) adapted from Figure 3 in the original article, and bottom shows results for the same parameter values in our implementation (b-d-f).

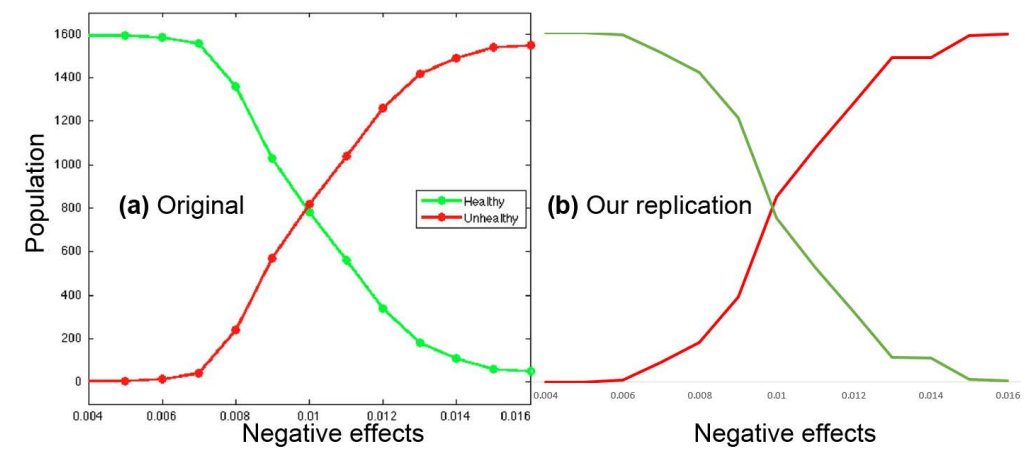

Figure 2: The original results (a) for a single run of the stochastic model have a comparable behavior to our replicated results (b).

To verify our implementation of the Schelling model, we compared the final (i) average level of similarity that agents have with their neighbors, computed as the average percentage of peers in an agent's neighborhood who share its state; and (ii) average percentage of agents that have no neighbors in a different state. In the comparison (Table 1), we note that numbers from the original articles (top row) were calculated by hand and reported as ratios rather than percentages (e.g., 2:1 instead of 33\% and 67\%). There was also limited information on the sample size or the distribution of outputs produced by the model (Schelling 1971). We thus compared with mean values (middle row) as well as 95\% Confidence Intervals (bottom row). By using the Confidence Intervals method to calculate the necessary number of runs for a 95\% CI (Robinson 2004), we established that 17 runs were necessary thus mean values are based on these runs. To ensure consistency, both the original and the replication were set to $50 \%$ demand and $70 \%$ density, with an even number of each population. 
Table 1: Verification of the Schelling model starting with initial populations of the same size.

\begin{tabular}{|l|l|l|} 
& Ending Similarity & $\%$ Surrounded \\
\hline Original & $90 \%$ & $67 \%$ \\
\hline Replication & $90 \%$ & $62 \%$ \\
\hline Replication CIs & {$[88.0 \%, 92.6 \%]$} & {$[59.3 \%, 65.5 \%]$}
\end{tabular}

Table 2: Verification of the Schelling model when varying the minority/majority ratio.

\begin{tabular}{|l|l|l|l|l|} 
& Minority Beginning & Majority Beginning & Minority Ending & Majority Ending \\
\hline Original & $33 \%$ & $67 \%$ & $67 \%$ & $80 \%$ \\
\hline Replication & $33 \%$ & $67 \%$ & $70 \%$ & $83 \%$ \\
\hline Replication CIs & {$[31.2 \%, 34.4 \%]$} & {$[64.9 \%, 68.1 \%]$} & {$[67.7 \%, 71.6 \%]$} & {$[82.7 \%, 84.9 \%]$}
\end{tabular}

In line with the original article, we also performed experiments exploring the model's outputs when starting with an uneven minority/majority ratio. Results consisting of the average level of similarity are shown in Table 2. This table is organized in the same manner as table 1, with original results in the top row and our averages/CIs following. The use of a $95 \% \mathrm{CI}$ resulted in a sample size of 37 runs.

\subsection{Experimental Design: Adding Uncertainty To The Original models}

We introduced a new parameter $U$ to both models, which conceptually represents the level of uncertainty agents have about the environment. For instance, in the eating model, agents now have a probability $U$ to make mistakes about the healthiness of their neighbors. This parameter was varied on the range $[0.00,0.50]$ by increments of .01 and runs were replicated enough times to generate $95 \%$ confidence intervals for each of the population counts at each level of uncertainty.

Thus, with the addition of $U$, the main input parameters of the Schelling model are tolerance/demand, uncertainty, initial density, and the ratio of majority to minority. With the addition of $U$ to the eating model, the main parameters are negative and positive influences $(N$ and $P)$, uncertainty, and purchasing power.

In the eating model, we measured the impact of $U$ by calculating the Pearson correlation for both the mean counts of each population, and the range of their confidence intervals (as a measure of variance). Our outcome variable was different for the Schelling model, owning to the model's focus on similarity as a final output (Tables 1-2). Consequently, we measure the impact of $U$ by measuring similarity for the Schelling model. We repeated this procedure for different levels of demand $(50 \%, 30 \%$, and $10 \%)$ to get a measure of how the Uncertainty parameter affects the outcome under different circumstances. All other parameters were left to their default values, such as a $70 \%$ density of agents and an even ratio of majority to minority.

\section{RESULTS}

\subsection{Eating model}

In line with the original study, we start by showing the outcomes of the model based on a single replication (Figure 3-a) across different levels of uncertainty. Although the visualization suggests that the value of $U$ (x-axis) does not impact the distribution of outcomes (y-axis), we note that there is variability across simulation runs. As exemplified in Figure 3-b for $U=0.2$, the prevalence of each state upon stabilization of the model can vary widely. To comprehensively assess whether errors in judgments impact outcomes, we thus examined $(i)$ the mean and (ii) the range for each state across replications. The number of replications 
was computed to achieve a 95\% Confidence Interval. Means and their ranges (defined by the span between upper and lower bounds) as shown in Figure 4 which confirms the initial suggestion that errors in judgment do not impact outcomes. We performed a statistical test for this hypothesis by computing the correlation between $U$ and both the mean and ranges.
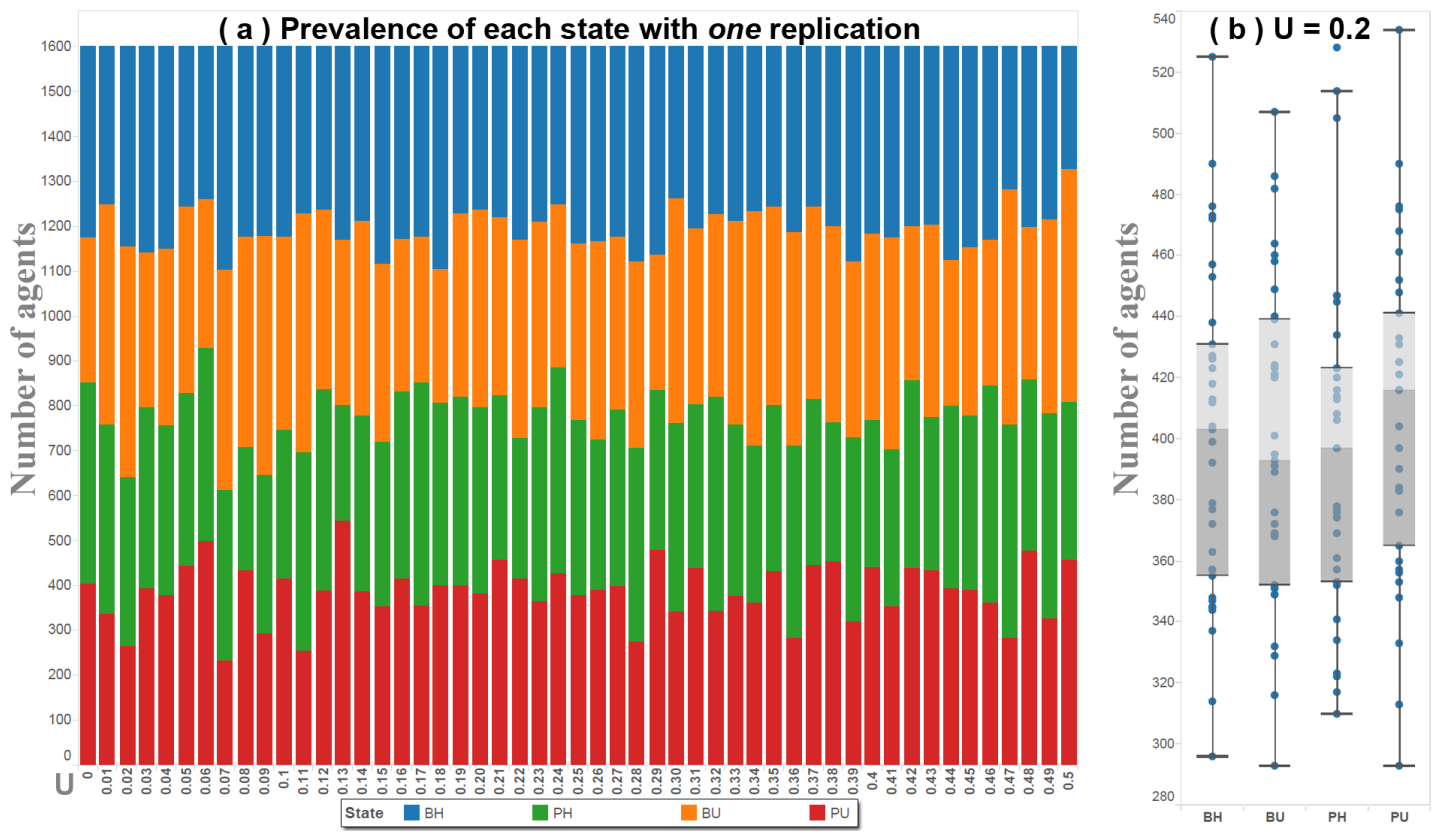

Figure 3: Sweep across $U$ on a single replication, as in the original article (a). Distribution of values using multiple replications, taking $U=0.2$ as example (b).

Based on the p-values summarized in Table 3, we found no significant correlations between the level of uncertainty and the mean values of each population $(p>.05)$ or the range of each confidence interval $(p>$ .05). Consequently, the model's outcomes do not change even as agents make an increasingly large number of mistakes in judging the health state of their peers. This result is unlike expectations in the real-world, hence it is possible that the model's design was not representing key aspects involved in decision-making.

Table 3: Testing for correlations between the uncertainty $U$ and the mean/range values for each state.

\begin{tabular}{|l|l|l|l|l|}
\hline State & Measure & Value & Correlation with uncertainty & p-value \\
\hline \multirow{2}{*}{ Purchase Unhealthy } & Mean & 398.03 & -.11 & .457 \\
\cline { 2 - 6 } & Range & 74.24 & -.05 & .717 \\
\hline \multirow{2}{*}{ Purchase Healthy } & Mean & 402.80 & -.02 & .908 \\
\cline { 2 - 5 } & Range & 78.40 & -.02 & .881 \\
\hline \multirow{2}{*}{ Bring Unhealthy } & Mean & 396.53 & .13 & .379 \\
\cline { 2 - 5 } & Range & 73.80 & .16 & .283 \\
\hline \multirow{2}{*}{ Bring Healthy } & Mean & 402.63 & -.01 & .954 \\
\cline { 2 - 5 } & Range & 70.84 & .04 & .776 \\
\hline
\end{tabular}

\subsection{Schelling model}

Given that higher demand results in higher similarity (section 3.1), our experimental design (section 3.2) examines the impact of a higher uncertainty across different levels of demand. Results in Figure 5 allow us 


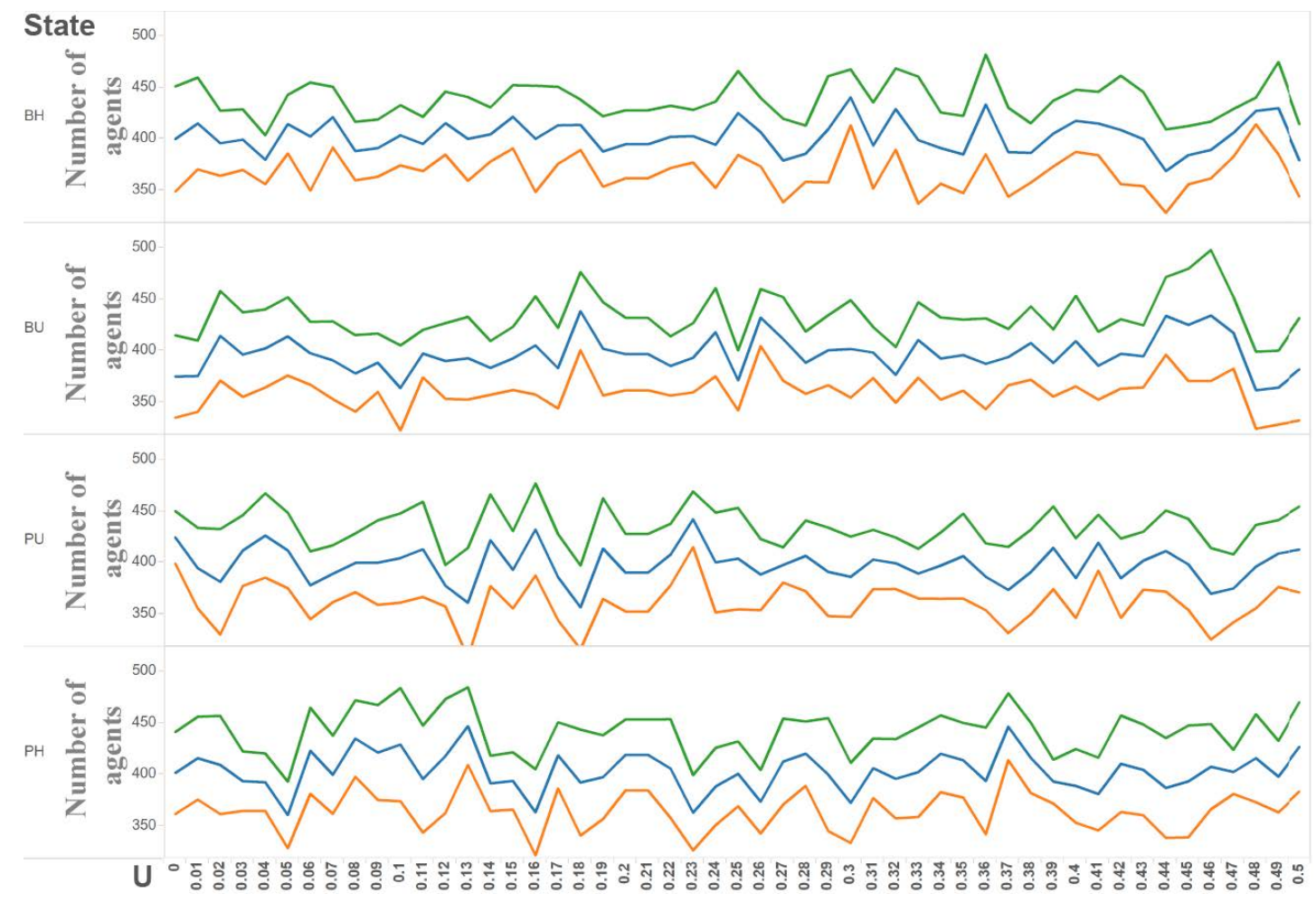

Figure 4: Average, upper, and lower bounds on the prevalence of each state across simulation runs for each value of the uncertainty parameter $U$.

to make two observations. First, uncertainty does have an effect on the output, unlike in the eating model. Specifically, higher uncertainty increases the stable similarity of the model. Second, the effect is mediated by demand: a lower level of demand is more impacted by uncertainty, but results in a lower maximum similarity (at $U=0.5$ ) than a higher level of demand. The impact of uncertainty also appears to plateau, with a set point depending on demands (e.g. $U=0.22$ for demand at $30 \%$ but $U=0.09$ for demand at $50 \%$ ).

In line with our investigation for the eating model, we used correlations to statistically examine the outcomes (y-axis in Figure 5) as a function of the uncertainty (x-axis). Table 3 demonstrates statistically significant correlations between errors in judgment and the outcome of the model. The trends present between correlations in different ranges of uncertainty (0-.1 vs. 0-.5) confirms that the correlation is generally stronger (meaning there is a more rapid transition in similarity) initially, and levels out as the model reaches higher levels of similarity with higher uncertainty.

\section{DISCUSSION AND CONCLUSION}

Simple Agent-Based Models of social influence consider that agents connected by a social tie will always influence each other, passing on perfect information (e.g., an agent can tell the state of another one) and making rational decisions. Some of these assumptions have been abandoned in various stands of ABM research, for instance using a similarity measure to drive "whether social influence occurs between connected individuals and how strongly they influence each other" (Flache et al. 2017). Such biases model that individuals prefer information confirming their ideas (similarity measure between the agents' mental models) or emanating from familiar peers (similarity measure between the agents' characteristics). Ultimately, including such biases can model the real-world phenomenon of individuals making judgment errors. 
Table 4: Similarity in the Schelling model across levels of uncertainty and demands. Each simulation ran until reaching a stable state.

\begin{tabular}{|l|l|l|l|}
\hline Demand level & Portion of dataset (i.e. values of U) & Correlation & Significance (i.e. p-value) \\
\hline \multirow{2}{*}{$10 \%$} & Whole (0 to 0.5) & .989 & $<.001$ \\
\cline { 2 - 4 } & Start (0 to 0.1) & .923 & $<.001$ \\
\hline \multirow{2}{*}{$30 \%$} & Whole (0 to 0.5) & .78 & $<.001$ \\
\cline { 2 - 4 } & Start (0 to 0.1) & .98 & $<.001$ \\
\hline \multirow{2}{*}{$50 \%$} & Whole (0 to 0.5) & .461 & .01 \\
\cline { 2 - 4 } & Start (0 to 0.1) & .940 & $<.001$ \\
\hline
\end{tabular}

Table 5: Assessing correlations across demand levels between uncertainty $(U)$ and population similarity.

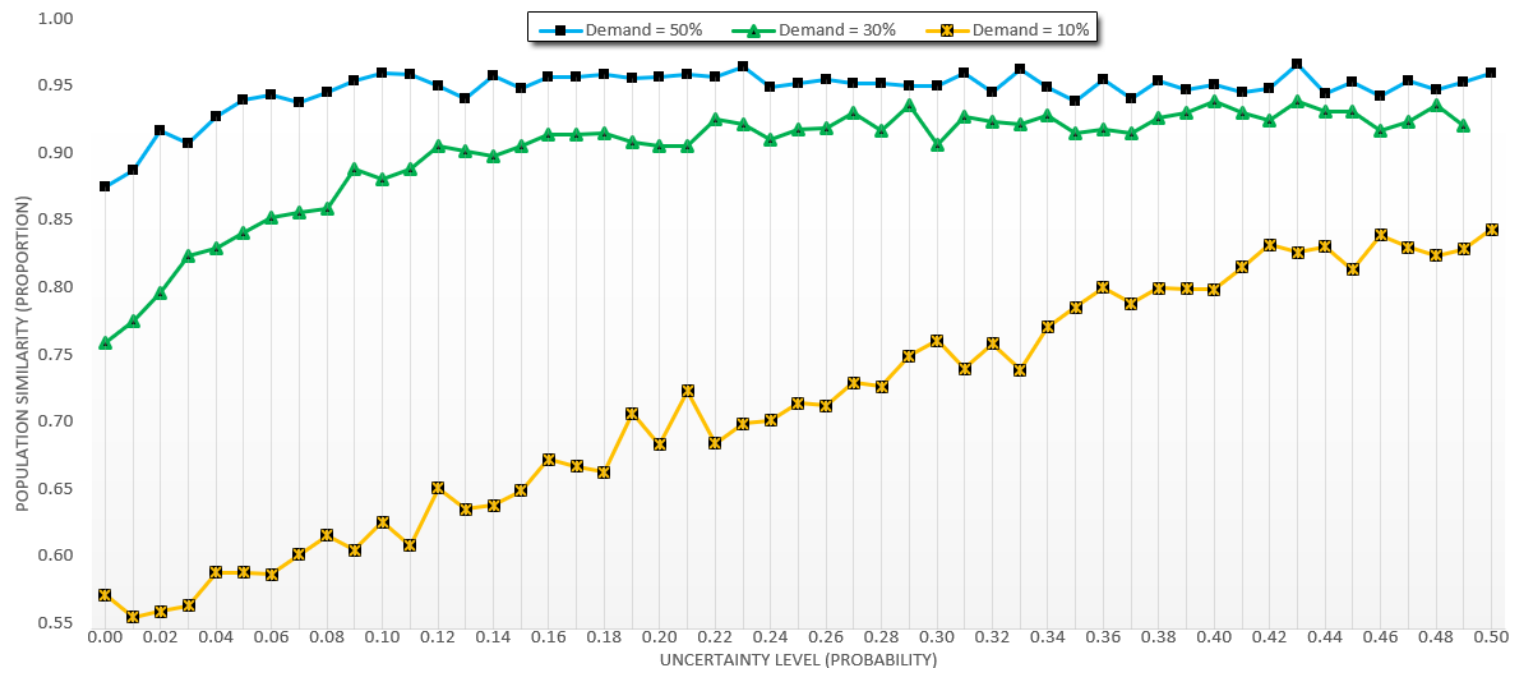

In this paper, we examined the implications of having agents incorrectly estimate the state of their peers. This differs from similarity measures, in which agents accurately know their traits or ideas such that they can compute similarities. It also differs from models in which manipulative agents purposely lie to derive benefits (Savarimuthu et al. 2011). Rather, we question the ability of agents to perfectly discriminate between the actions of others or agree on a population-wide definition of states. For instance, the healthy eating model that we revisited initially assumed that an agent could clearly tell whether a peer engages in healthy or unhealthy eating. Although definitions vary, 'unhealthy' foods usually covers items whose high amount of calories is primarily derived from fat or sugar. An agent may see a peer eating salads and follow this seemingly healthy behavior by buying the same salads, without knowing that the dressing is sweeter than eight donuts or that crumbs and bacon contain more calories than several slices of pizza. Studies have shown that individuals incorrectly judge foods based on the body size of the peer eating them. For instance, seminal experimental studies noted that having a slim peer eat a large amount of food resulted in participants eating $70 \%$ to $241 \%$ more snacks - a greater effect than observed with overweight peers (McFerran et al. 2009). Our study thus contributes to shedding light on the dynamics of social influence in ABMs by including perceptual uncertainty to mimic the inaccurate judgments observed in reality.

Although experimental studies show that our eating decisions are partly driven by misconceptions, we found that adding such incorrect judgments to the eating model had no effect on the outcome. Even a massive level of errors did not change how agents transitioned between states, as evidenced by the absence of a correlation between the level of errors in judgment and either the mean or range of agents in each state. In contrast, the Schelling segregation model resulted in a more nuanced picture: at low levels of demand, a rise in errors 
has a linear impact on the model outcomes whereas at higher levels of demands the effect reaches a plateau. Consequently, our main finding is that the effect of making errors in judgment strongly depends on the design of a model. In other words, the decision-making component of some models may not be as sensitive to judgment errors as its real-world counterpart, while other models have more built-in flexibility. Further research is needed to make a model more sensitive to agent uncertainty (thus being closer to real-world conditions) without being more vulnerable to perturbations in general (thus decreasing its robustness).

Generalizing our findings would require more models. The limiting factor is the lack of disclosure into the code base of published models. Since $90 \%$ of ABM studies do not release their code (Janssen 2017), we need to re-implement the models (often based on narratives) and verify the implementations by comparing figures or summary tables (since output files are rarely available). While we examined other models, our implementations did not produce the authors' original figures or tables, potentially due to ambiguity in the model's description or unknown behaviors in libraries used by the authors. Comprehensively assessing the effects of increasing the realism in the decision-making module of agents will thus benefit from the transparency and replication efforts underway in the modeling community. An emerging research avenue is to navigate the growing ABM code repositories (e.g., https://www.comses.net/) to identify well-documented models (Vendome et al. 2020) that include social interactions and lack perceptual uncertainty.

\section{CONTRIBUTIONS}

This project was designed and supervised by PJG. EOG programmed the models, performed the experiments, and analyzed the results. EOG and PJG wrote the manuscript.

\section{ACKNOWLEDGEMENTS}

EOG thanks Furman University and its Department of Computer Science for financial support, both through The Furman Advantage and departmental funds. PJG also thanks the Department of Computer Science for providing a high-performance workstation to perform experiments.

\section{REFERENCES}

Asch, S. E. 1955. “Opinions and Social Pressure”. Scientific American vol. 193 (5), pp. 31-35.

Badham, J., E. Chattoe-Brown, N. Gilbert, Z. Chalabi, F. Kee, and R. F. Hunter. 2018. "Developing agentbased models of complex health behaviour". Health \& place vol. 54, pp. 170-177.

Bahr, D. B., R. C. Browning, H. R. Wyatt, and J. O. Hill. 2009. "Exploiting Social Networks to Mitigate the Obesity Epidemic". Obesity vol. 17 (4), pp. 723-728.

Bianchi, F., and F. Squazzoni. 2015. "Agent-based models in sociology". Wiley Interdisciplinary Reviews: Computational Statistics vol. 7 (4), pp. 284-306.

Brashears, M., M. Konner, C. Madsbjerg, L. McNamara, and K. Sieck. 2019. Moving Social-Behavioral Modeling Forward: Insights from Social Scientists, Chapter 12, pp. pp. 263-277. John Wiley \& Sons.

Burden, D., and M. Savin-Baden. 2019. Mind, pp. 71-96. CRC Press.

Carey, G., E. Malbon, N. Carey, A. Joyce, B. Crammond, and A. Carey. 2015. "Systems science and systems thinking for public health: a systematic review of the field". BMJ open vol. 5 (12), pp. e009002.

Chattoe-Brown, E. 2013. "Why sociology should use agent based modelling". Sociological Research Online vol. 18 (3), pp. 1-11. 
Dabbaghian, V., V. K. Mago, T. Wu, C. Fritz, and A. Alimadad. 2012. "Social interactions of eating behavior among high school students: a cellular automata approach". BMC Medical Research Methodology vol. 12 (1), pp. 155.

Dabbaghian, V., V. Spicer, S. Singh, P. Borwein, and P. Brantingham. 2011. "The social impact in a high-risk community: A cellular automata model”. J Comput Sci vol. 2 (3), pp. 238-246.

dos Santos, R. M. Z., and S. Coutinho. 2001. "Dynamics of HIV infection: A cellular automata approach". Physical review letters vol. 87 (16), pp. 168102.

Echzell, H., T. Friedrich, P. Lenzner, L. Molitor, M. Pappik, F. Schöne, F. Sommer, and D. Stangl. 2019. Convergence and hardness of strategic schelling segregation, pp. 156-170.

Evans, A. 2012. "Uncertainty and error”. In Agent-based models of geographical systems, pp. 309-346. Springer.

Ferreira, M. I. A. 2019. The Dialectics of Agent/Environment, pp. 1-12. Springer Int. Publishing.

Flache, A., M. Mäs, T. Feliciani, E. Chattoe-Brown, G. Deffuant, S. Huet, and J. Lorenz. 2017. "Models of social influence: Towards the next frontiers". J. of Artificial Societies and Social Simulation vol. 20 (4).

Giabbanelli, P., and R. Crutzen. 2013. "An agent-based social network model of binge drinking among Dutch adults". J. of Artificial Societies and Social Simulation vol. 16 (2), pp. 10.

Giabbanelli, P. J. 2019. Solving Challenges at the Interface of Simulation and Big Data Using Machine Learning, pp. 572-583.

Giabbanelli, P. J., A. Alimadad, V. Dabbaghian, and D. T. Finegood. 2012. "Modeling the influence of social networks and environment on energy balance and obesity". J Comput Sci vol. 3 (1-2), pp. 17-27.

Giabbanelli, P. J., and R. Crutzen. 2017. "Using agent-based models to develop public policy about food behaviours: Future directions and recommendations". Computational and mathematical methods in medicine vol. 2017.

Giabbanelli, P. J., C. Freeman, J. A. Devita, N. Rosso, and Z. L. Brumme. 2019. Mechanisms for Cell-to-cell and Cell-free Spread of HIV-1 in Cellular Automata Models, pp. 103-114.

Hassan, S., L. Garmendia, and J. Pavón. 2010. "Introducing uncertainty into social simulation: using fuzzy logic for agent-based modelling”. Int. J. of Reasoning-Based Intelligent Systems vol. 2 (2), pp. 118-124.

Immorlica, N., R. Kleinbergt, B. Lucier, and M. Zadomighaddam. 2017. Exponential segregation in a twodimensional schelling model with tolerant individuals, pp. 984-993.

Janssen, M. A. 2017. "The practice of archiving model code of agent-based models". Journal of Artificial Societies and Social Simulation vol. 20 (1).

Kadushin, C. 2012a. Networks as Social Capital, pp. 162-181. Oxford University Press.

Kadushin, C. 2012b. Networks, Influences, and Social Diffusion, pp. 135-159. Oxford University Press.

Khademi, A., D. Zhang, P. J. Giabbanelli, S. Timmons, C. Luo, and L. Shi. 2018. "An Agent-Based Model of Healthy Eating with Applications to Hypertension". In Advanced Data Analytics in Health, pp. 43-58. Springer.

Klein, D., J. Marx, and K. Fischbach. 2018. "Agent-Based Modeling in Social Science, History, and Philosophy. An Introduction”. Historical Social Research vol. 43 (1 (163), pp. 7-27.

Mabry, P. L., S. E. Marcus, P. I. Clark, S. J. Leischow, and D. Méndez. 2010. "Systems science: a revolution in public health policy research". American J. of Public Health vol. 100, pp. 1161-1163.

Maglio, P. P., and P. L. Mabry. 2011. "Agent-based models and systems science approaches to public health". American J. of preventive medicine vol. 40 (3), pp. 392-394.

Masad, D., and J. Kazil. 2015. MESA: an agent-based modeling framework, pp. 53-60. 
McFerran, B., D. W. Dahl, G. J. Fitzsimons, and A. C. Morales. 2009. "I'll have what she's having: Effects of social influence and body type on the food choices of others". J. Consum. Res. vol. 36 (6), pp. 915-929.

Nianogo, R. A., and O. A. Arah. 2015. "Agent-based modeling of noncommunicable diseases: a systematic review". American J. of public health vol. 105 (3), pp. e20-e31.

Nyblade, B., A. O'Mahony, and K. Sieck. 2019. Building on Social Science: Theoretic Foundations for Modelers, Chapter 4, pp. pp. 63-99. John Wiley \& Sons.

Parker, J., and J. M. Epstein. 2011. "A distributed platform for global-scale agent-based models of disease transmission". ACM Transactions on Modeling and Computer Simulation (TOMACS) vol. 22 (1), pp. 2.

Railsback, S. F., and V. Grimm. 2010. Models, Agent-Based Models, and the Modeling Cycle, pp. 3-13. Princeton University Press.

Robinson, S. 2004. Simulation: The Practice of Model Development and Use. John Wiley \& Sons.

Russell, Nestar John Charles 2011. "Milgram's obedience to authority experiments: Origins and early evolution".

Savarimuthu, B. T. R., R. Arulanandam, and M. Purvis. 2011. Aspects of active norm learning and the effect of lying on norm emergence in agent societies, pp. 36-50.

Schelling, T. C. 1971. "Dynamic models of segregation”. J Math Sociol vol. 1 (2), pp. 143-186.

Simon, H. 1957. "A behavioral model of rational choice". Models of man, social and rational: Mathematical essays on rational human behavior in a social setting, pp. 241-260.

Spicer, V., A. A. Reid, J. Ginther, H. Seifi, and V. Dabbaghian. 2012. "Bars on blocks: A cellular automata model of crime and liquor licensed establishment density". Computers, Environment and Urban Systems vol. 36 (5), pp. $412-422$.

Tversky, A., and D. Kahneman. 1974. "Judgment under uncertainty: Heuristics and biases". Science vol. 185 (4157), pp. 1124-1131.

Urselmans, L., and S. Phelps. 2018. "A Schelling model with adaptive tolerance”. PloS one vol. 13 (3), pp. e0193950.

Vendome, C., D. M. Rao, and P. J. Giabbanelli. 2020. How do modelers code artificial societies? Investigating practices and quality of NetLogo codes from large repositories.

Wilensky, U., and W. Rand. 2015. Why Agent Based Modeling?, pp. 1-20. MIT Press.

Zhang, D., P. J. Giabbanelli, O. A. Arah, and F. J. Zimmerman. 2014. "Impact of different policies on unhealthy dietary behaviors in an urban adult population: an agent-based simulation model". American J. of public health vol. 104 (7), pp. 1217-1222.

\section{AUTHOR BIOGRAPHIES}

ETHAN O. GRANTHAM is a student in computer science and psychology at Furman University (USA). His research interests are at the interface of cognitive psychology and computer science. His research was supported by the Furman Advantage and the Computer Science Department at Furman University, while supervised by Dr. Giabbanelli. His email address is ethan.grantham@furman.edu.

PHILIPPE J. GIABBANELLI, Ph.D., is an Associate Professor in the Department of Computer Science \& Software Engineering at Miami University (USA). His research interests include network science, machine learning, and simulation. He currently serves as track chair for the 2020 Spring Simulation Conference, and program chair for the 2020 ACM SIGSIM Principles of Advanced Discrete Simulations (PADS) conference. His email address is giabbapj@miamioh.edu. His website is https://www.dachb.com. 